\title{
On the methodologies for the assessment of the impact of parameters in acoustophoretic separation devices
}

\author{
Fabio Garofalo ${ }^{1}[\mathbb{D}$
}

Received: 2 December 2018 / Accepted: 13 May 2019 / Published online: 11 June 2019

(c) The Author(s) 2019

\begin{abstract}
In this communication, we reconcile the kinematic method illustrated by some authors (Yang et al. in Microfluid Nanofluid 22:44-56, 2018; Vitali et al. in RSC Adv 8:38955, 2018) in studying the impact of system and suspension parameters on acoustophoretic separations with the statistical method formerly proposed by Garofalo (Microfluid Nanofluid 18(3):367382, 2014a; ASME 2014 3rd global congress on nanoengineering for medicine and biology NEMB2014-93092, 2014b. https://www.researchgate.net/publication/259962346_Free-flow_acoustofluidic_devices_kinematics_cross-sectional_dispe rsion_and_particle_ensemble_correlations_Presentation) and lately extended to particle populations by the same author (Garofalo in CBMS the 14th conference on acoustofluidics, San Diego (CA), August 28-29, 2017, 2017. https://www.resea rchgate.net/publication/259962346_Free-flow_acoustofluidic_devices_kinematics_cross-sectional_dispersion_and_parti cle_ensemble_correlations_Presentation; Quantifying acoustophoretic separation of microparticle populations by meanand-covariance dynamics for Gaussians in mixture models, 2018. arXiv:1802.09790). The connection between these two methods is established by (1) reinterpreting the kinematic method in terms of tangent space dynamics, and (2) transforming the dynamics in the tangent space into the dynamics of the area elements. The dynamics of the area elements is equivalent to the dynamics of the covariance matrix derived by moment analysis and associated with the dispersion problem during microparticle acoustophoresis. The similarities and the differences between the kinematic based method and the stochastic method proposed by the present author are illustrated and discussed in the light of the numerical results for a prototypical model of acoustophoretic separation.
\end{abstract}

Keywords Acoustofluidics · Particle separation · Acoustic standing waves

\section{Introduction}

Some authors (Yang et al. 2018; Vitali et al. 2018) have investigated the influence of the particle and system parameters on the acoustophoretic separation of microparticles by considering the integration of the equation of motion for a particle suspended in a medium

$\frac{\mathrm{d} \boldsymbol{x}}{\mathrm{d} t}=\boldsymbol{v}(\boldsymbol{x}, \boldsymbol{p}), \quad \boldsymbol{x}(0)=\boldsymbol{x}_{0}$,

where $\boldsymbol{x}=[x, y, z]^{\mathrm{T}}$ is the particle position in the threedimensional space, $\boldsymbol{x}_{0}=\left[x_{0}, y_{0}, z_{0}\right]^{\mathrm{T}}$ the initial position, and $\boldsymbol{p}=\left[p_{1}, \ldots, p_{N}\right]^{\mathrm{T}}$ is a set of $N$ parameters. This set includes

Fabio Garofalo

fabio.garofalo@bme.lth.se

1 Department of Biomedical Engineering, Lund University, Ole Römers Väg 3, 22363 Lund, Sweden all the parameters that matter in quantifying the acoustophoretic separation: particle properties, fluid properties, and device properties. Yang et al. subdivided the parameters in intrinsic and extrinsic, this subdivision is not used here.

Since in Yang et al. (2018), Vitali et al. (2018) the authors aimed to develop the analysis for quantifying the separation of biological samples, they suggested, by using the words "dispersed objects", "populations" or "average position", that the statistics of the sample was taken into account to some extent. However, in their paper they did not consider any statistical analysis of dispersion during the dynamics of the separation process. Rather, they considered two (or more) extremal values for the initial positions or parameters and studied the deviation of two trajectories corresponding to the "worst" and "best" cases. This led to a troublesome analysis [see Eq. (9) in Vitali et al. (2018)] where the number of equations is quite large. However, the problem of studying the influence of small deviations from initial conditions is well known in dynamical 
system theory. Furthermore, founding their approach on this kind of methodology, they heuristically defined indicators to quantify the separation, such as the "relative displacement", the "ideal separation efficiency", and the "separation efficiency". These indicators are functions based on the distances along the separation direction, i.e., the $y$-direction, between the kinematic trajectories resulting from the integration of equation of motion for the extremal values of the particle/system parameters.

Furthermore, it must be noticed that in citing Garofalo (2014a) and by addressing that "the theoretical analysis of particle kinematics in free-flow acoustophoretic devices was already reported", they definitively overlooked the opportunity to discuss the results of their study of dispersion against a seemingly different method for the study of the "cross-sectional dispersion". Indeed, the similarity between the behavior of the bandwidth (Yang et al. 2018; Vitali et al. 2018) and that of the variance formerly presented in Garofalo (2014a) and successively in Garofalo $(2017,2018)$ is noticeable. This affinity goes beyond the bandwidth and the variance when one compares the graphs of the trajectories/bandwidth in Yang et al. (2018) and those for the spatial distributions in Garofalo (2014a). This link went unnoticed and missing in the discussion of the results presented by the authors in Yang et al. (2018), Vitali et al. (2018).

This communication aims to compare and reconcile the approach in Yang et al. (2018), Vitali et al. (2018) with the statistic-based method for studying dispersion during acoustophoretic separation proposed by Garofalo (2014a, b, 2017, 2018).

\section{Theoretical comparison}

\subsection{Dynamics}

Let us consider the extension of Eq. (1) in the coordinate and parameter space by including a dummy dynamics in the parameters, i.e., $\boldsymbol{p}(t)=\boldsymbol{p}_{0}$,

$\frac{\mathrm{d} x}{\mathrm{~d} t}=\boldsymbol{v}(\boldsymbol{x}, \boldsymbol{p}), \quad \boldsymbol{x}(0)=x_{0}$,

$\frac{\mathrm{d} \boldsymbol{p}}{\mathrm{d} t}=\mathbf{0}, \quad \boldsymbol{p}(0)=\boldsymbol{p}_{0}$.

These equations can be rewritten in the space-parameter coordinates by introducing $\boldsymbol{m}=\left[\boldsymbol{m}^{x}, \boldsymbol{m}^{p}\right]^{\mathrm{T}}=[\boldsymbol{x}, \boldsymbol{p}]^{\mathrm{T}}$ and the drift $\boldsymbol{f}=[\boldsymbol{v}, \mathbf{0}]^{\mathrm{T}}$

$\frac{\mathrm{d} \boldsymbol{m}}{\mathrm{d} t}=\boldsymbol{f}(\boldsymbol{m}), \quad \boldsymbol{m}(0)=\boldsymbol{m}_{0}$,

Let us note that Eq. (2), or its compact version Eq. (3), corresponds to the very specific case of constant parameters.
While this in general is true, there can be situations where this assumption is not valid. For example, as the particle moves in an inhomogeneous fluid, the physical parameters can have an implicit dependence on the particle position and vary during the motion of the particle along the trajectory; it results in $\boldsymbol{p}=\boldsymbol{p}(\boldsymbol{x})$.

The essence of the Yang's method is to consider the dynamics of Eq. (4) for two perturbations with initial distance $\boldsymbol{u}(0)=\boldsymbol{u}_{0}$, namely

$\frac{\mathrm{d} \boldsymbol{m}_{-}}{\mathrm{d} t}=\boldsymbol{f}\left(\boldsymbol{m}_{-}\right), \quad \boldsymbol{m}_{-}(0)=\boldsymbol{m}_{0}-\frac{1}{2} \boldsymbol{u}_{0}$,

$\frac{\mathrm{d} \boldsymbol{m}_{+}}{\mathrm{d} t}=\boldsymbol{f}\left(\boldsymbol{m}_{+}\right), \quad \boldsymbol{m}_{+}(0)=\boldsymbol{m}_{0}+\frac{1}{2} \boldsymbol{u}_{0}$.

where $\boldsymbol{u}_{0}=\mathrm{BW}(0) \hat{\boldsymbol{y}}+\boldsymbol{u}_{0}^{p}$ corresponds to the initial value of the bandwidth BW along the $y$-direction plus the parameters' perturbation $\boldsymbol{u}_{0}^{p}$ with $\boldsymbol{u}_{0}^{p} \cdot \hat{\boldsymbol{y}}=0$. Half-summing and subtracting Eqs. (4) and (5), expanding up to the first order $f$ in the small perturbation $\boldsymbol{u}$ around $\boldsymbol{m}$, and resulting in this case $\boldsymbol{m} \simeq \frac{1}{2}\left(\boldsymbol{m}_{+}+\boldsymbol{m}_{-}\right)$and $\boldsymbol{u} \simeq \boldsymbol{m}_{+}-\boldsymbol{m}_{-}$, Eqs. (4) and (5) result equivalent to

$\frac{\mathrm{d} \boldsymbol{m}}{\mathrm{d} t}=\boldsymbol{f}(\boldsymbol{m}), \quad \boldsymbol{m}(0)=\boldsymbol{m}_{0}$,

$\frac{\mathrm{d} \boldsymbol{u}}{\mathrm{d} t}=\boldsymbol{J}(\boldsymbol{m}) \cdot \boldsymbol{u}, \quad \boldsymbol{u}(0)=\boldsymbol{u}_{0}$.

where $\boldsymbol{J}(\boldsymbol{m})=\boldsymbol{\nabla} \boldsymbol{f}(\boldsymbol{m})$ is the Jacobian of the drift computed in the correspondence of $\boldsymbol{m}$. Noting that Eq. (6b) can be rewritten in Einstein notation $(h=1 \ldots N+3)$

$\frac{\mathrm{d} u^{h}}{\mathrm{~d} t}=\partial_{\curlywedge} f^{h}(\boldsymbol{m}) u^{l}, \quad u^{h}(0)=u_{0}^{h}$,

introducing the Kronecker product of the perturbation components $s^{h k}=u^{h} u^{k}$, and considering that its time derivative is

$\frac{\mathrm{d} s^{h k}}{\mathrm{~d} t}=\frac{\mathrm{d} u^{h}}{\mathrm{~d} t} u^{k}+u^{h} \frac{\mathrm{d} u^{k}}{\mathrm{~d} t}$,

in place of the Eqs. (6), the system

$\frac{\mathrm{d} \boldsymbol{m}}{\mathrm{d} t}=\boldsymbol{f}(\boldsymbol{m}), \quad \boldsymbol{m}(0)=\boldsymbol{m}_{0}$,

$\frac{\mathrm{d} \boldsymbol{s}}{\mathrm{d} t}=\boldsymbol{J}(\boldsymbol{m}) \cdot \boldsymbol{s}+\boldsymbol{s} \cdot \boldsymbol{J}^{T}(\boldsymbol{m}), \quad \boldsymbol{s}(0)=\boldsymbol{s}_{0}$,

can be considered, in which $\boldsymbol{s}_{0}=\boldsymbol{u}_{0} \boldsymbol{u}_{0}^{T}$ is the initial value for $\boldsymbol{s}$. Note that, the covariance matrix is $(3+N) \times(3+N)$ symmetric matrix

$s=\left(\begin{array}{cc}s^{x x} & \left(s^{x p}\right)^{T} \\ s^{x p} & s^{p p}\end{array}\right)$ 
where $s^{x x}$ is a $3 \times 3$ matrix, $s^{p p}$ is an $N \times N$ matrix, and $s^{x p}$ is an $N \times 3$ matrix. Note also that for the parameter components results, $\frac{\mathrm{d}}{\mathrm{d} t} \boldsymbol{m}^{p}=\mathbf{0}$ and $\frac{\mathrm{d}}{\mathrm{d} t} s^{p \boldsymbol{p}}=\mathbf{0}$.

Equations (9) are the multivariate and diffusion-less form of equations used for studying dispersion in Garofalo (2014a), and extended to microparticle populations with arbitrary statistics that is equivalent to large perturbations, in Garofalo (2017, 2018). Equations (9) have been addressed as mean-and-covariance dynamics in Garofalo (2018), where also their connection with stochastic linearization methods has been recognized (see reference therein).

\subsection{Indicators}

The knowledge of the mean and the covariance by solution of Eqs. (9) enables for the approximation of the dynamics for the probability density function (PDF)

$\rho(\boldsymbol{q}, t)=\mathcal{N}[\boldsymbol{q} \mid \boldsymbol{m}(t), \boldsymbol{s}(t)]$,

where $\mathcal{N}(\boldsymbol{q} \mid \boldsymbol{m}, \boldsymbol{s})$ is a multivariate normal distribution with mean $\boldsymbol{m}$ and covariance matrix $\boldsymbol{s}$. Most relevant for the present discussion is the spatial marginal

$\rho^{\text {spatial }}(x, t)=\mathcal{N}\left[\boldsymbol{x} \mid \boldsymbol{m}^{x}(t), s^{x x}(t)\right]$,

which with a suitable parametrization, e.g., in the $x$-direction, enables the introduction of indicators similar to those addressed by Yang and used by Vitali.

Bandwidth From the derivation of the dynamics of the covariance $\boldsymbol{s}$ by the dynamics of $\boldsymbol{u}$ in the tangent space, we infer that the bandwidth BW is comparable to the square root of the variance in the $y$-direction

$\mathrm{BW}(\xi)=\left|\left[\boldsymbol{m}_{+}(\xi)-\boldsymbol{m}_{-}(\xi)\right] \cdot \hat{\boldsymbol{y}}\right| \simeq \sqrt{s^{y y}}(\xi)$,

where $\xi$ is a generic coordinate corresponding to the "section" where the comparison is performed.

Separation efficiencies A quantity similar to the Yang's ideal separation efficiency ISE can be derived from the statistical method by computing the integral along the $y$-direction of the product of two spatial marginals at a given section $\xi$ Garofalo (2014b). The inverse of the separation resolution is

$$
\begin{aligned}
\operatorname{SR}^{-1}(\xi) & =\int_{0}^{H} \rho_{1}^{\text {spatial }}(\xi, y) \rho_{2}^{\text {spatial }}(\xi, y) \mathrm{d} y \\
& \simeq \int_{-\infty}^{\infty} \rho_{1}^{\text {spatial }}(\xi, y) \rho_{2}^{\text {spatial }}(\xi, y) \mathrm{d} y
\end{aligned}
$$

and this integral admits an analytical solution in the case when the marginals are Gaussians
$\mathrm{SR}^{-1}(\xi)=\frac{\exp \left\{-\frac{\left[m_{1}^{y}(\xi)-m_{2}^{y}(\xi)\right]^{2}}{2\left[s_{1}^{y y}(\xi)+s_{2}^{y y}(\xi)\right]}\right\}}{\sqrt{2 \pi\left[s_{1}^{y y}(\xi)+s_{2}^{y y}(\xi)\right]}}$.

Note the similitude between the square root of the exponential argument, here named resolution index RI, and the Yang's ISE

$\operatorname{RI}(\xi)=\frac{\left|m_{1}^{y}(\xi)-m_{2}^{y}(\xi)\right|}{\sqrt{2}\left[s_{1}^{y y}(\xi)+s_{2}^{y y}(\xi)\right]^{1 / 2}}$,

$\operatorname{ISE}(\xi)=2 \frac{\left|\left\langle y_{1}\right\rangle(\xi)-\left\langle y_{2}\right\rangle(\xi)\right|}{\mathrm{BW}_{1}(\xi)+\mathrm{BW}_{2}(\xi)}$,

where $\langle y\rangle=\frac{1}{2}\left(\boldsymbol{m}_{+}+\boldsymbol{m}_{-}\right) \cdot \hat{\boldsymbol{y}}$. Finally, the Yang's separation efficiency SE is essemptially the ideal separation efficiency ISE with a radius correction. There is no equivalent/similar quantity in the statistical approach proposed by Garofalo (2018). Furthermore, some issues arise when this quantity is analyzed, as the separation efficiency SE is defined only for particles with the same radii, and this induces some limitations in the method used by Yang and Vitali. Conversely, the statistics-based method considers the dependence of the dispersion properties on the coordinates and parameters on equal footing, embedding all these contributions in the dynamics of $s$ and, as a consequence, their impact is encoded in both SR and RI (Fig. 1).

\section{Results}

To show the quantitative comparison of the two approaches while performing effortless analytical calculations, let us consider the following prototypical model for acoustophoresis reported in Section III.A of Garofalo (2018)

$v(y, r)=\alpha r^{2} \sin (2 \pi y)$,

for which the mean-and-covariance dynamics results are

$\frac{\mathrm{d} m^{y}}{\mathrm{~d} t}=\alpha\left(m_{0}^{r}\right)^{2} \sin \left(2 \pi m^{y}\right)$,

$\begin{aligned} \frac{\mathrm{d} s^{y r}}{\mathrm{~d} t}= & \alpha\left[2 \pi\left(m_{0}^{r}\right)^{2} \cos \left(2 \pi m^{y}\right) s^{y r}\right. \\ & \left.+2 m_{0}^{r} \sin \left(2 \pi m^{y}\right) s_{0}^{r r}\right],\end{aligned}$

$\begin{aligned} \frac{\mathrm{d} s^{y y}}{\mathrm{~d} t}= & \alpha\left[4 \pi\left(m_{0}^{r}\right)^{2} \cos \left(2 \pi m^{y}\right) s^{y y}\right. \\ & \left.+4 m_{0}^{r} \sin \left(2 \pi m^{y}\right) s^{y r}\right],\end{aligned}$ 

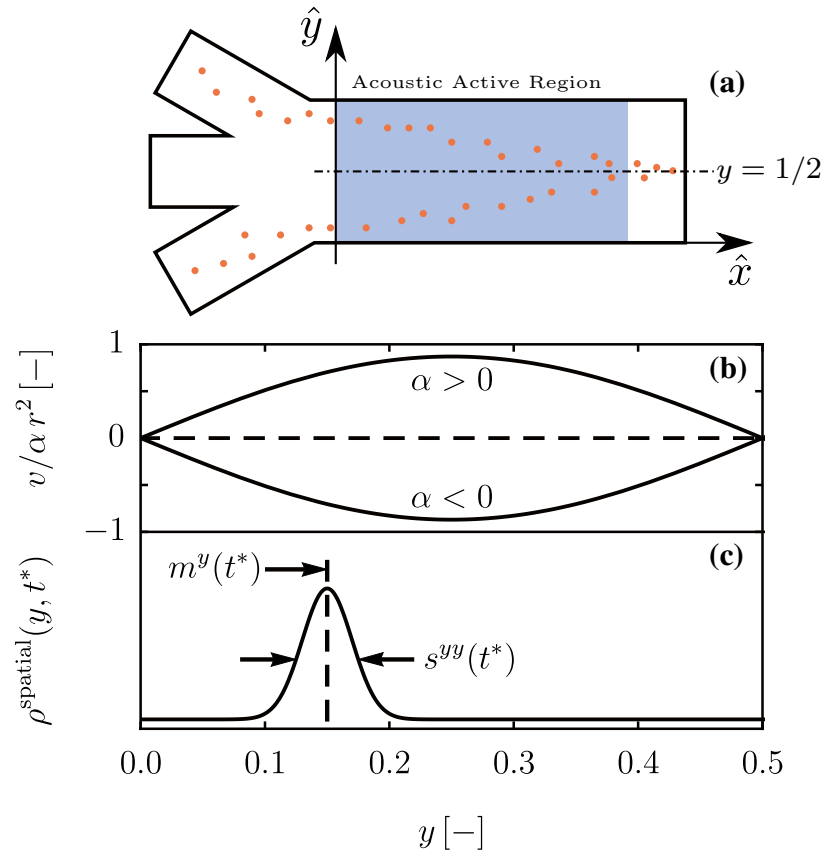

Fig. 1 (Color Online) a Top view schematic of a separation device, and one-dimensional prototypical model: $\mathbf{b}$ dimensionless velocity $v / \alpha r^{2}$ for $\alpha>0$ and $\alpha<0$, i.e., Eq. (18), and c spatial marginal for time $t=t^{*}$

where $m_{0}^{r}$ and $s_{0}^{r r}$ are the mean radius and its variance, respectively. Figure 6 shows (A) the schematic of the separation device, (B) the dimensionless velocity field, and (C) an example of the spatial marginal $\rho^{\text {spatial }}(y, t)$ for time $t=t^{*}$. The initial conditions for Eqs. (19) are chosen so as to compare the bandwidth areas and the dispersion bands in Garofalo's method (we consider for all the cases $s^{y r}(0)=0$, and $\alpha=1$ except when the comparison between the indicators is considered).

Figure 2a shows the comparison between the two methods for different values of the inlet condition $y_{0}$, a relative initial perturbation equals to $\mathrm{BW}(0)=\sqrt{s_{0}^{y y}}=50 \% y_{0}$, radius $m^{r}=1$, and no initial perturbation on the radius $s^{r r}=0$. Panel (A) reports the comparison between the bandwidth areas $y \in\left[y_{-}, y_{+}\right]$and the dispersion bands $m^{y} \pm \frac{1}{2} \sqrt{s^{y y}}$. It can be seen that the results for the two methods almost coincide, and for quantifying the small differences between the two methods we introduce the distances

$\Delta_{\text {traj }}(t)=\frac{\left|m^{y}(t)-\langle y\rangle(t)\right|}{m^{y}(t)}$,

for the trajectory, and

$\Delta_{\text {disp }}(t)=\frac{\left|\sqrt{s^{y y}(t)}-\mathrm{BW}(t)\right|}{1+\sqrt{s^{y y}(t)}}$,
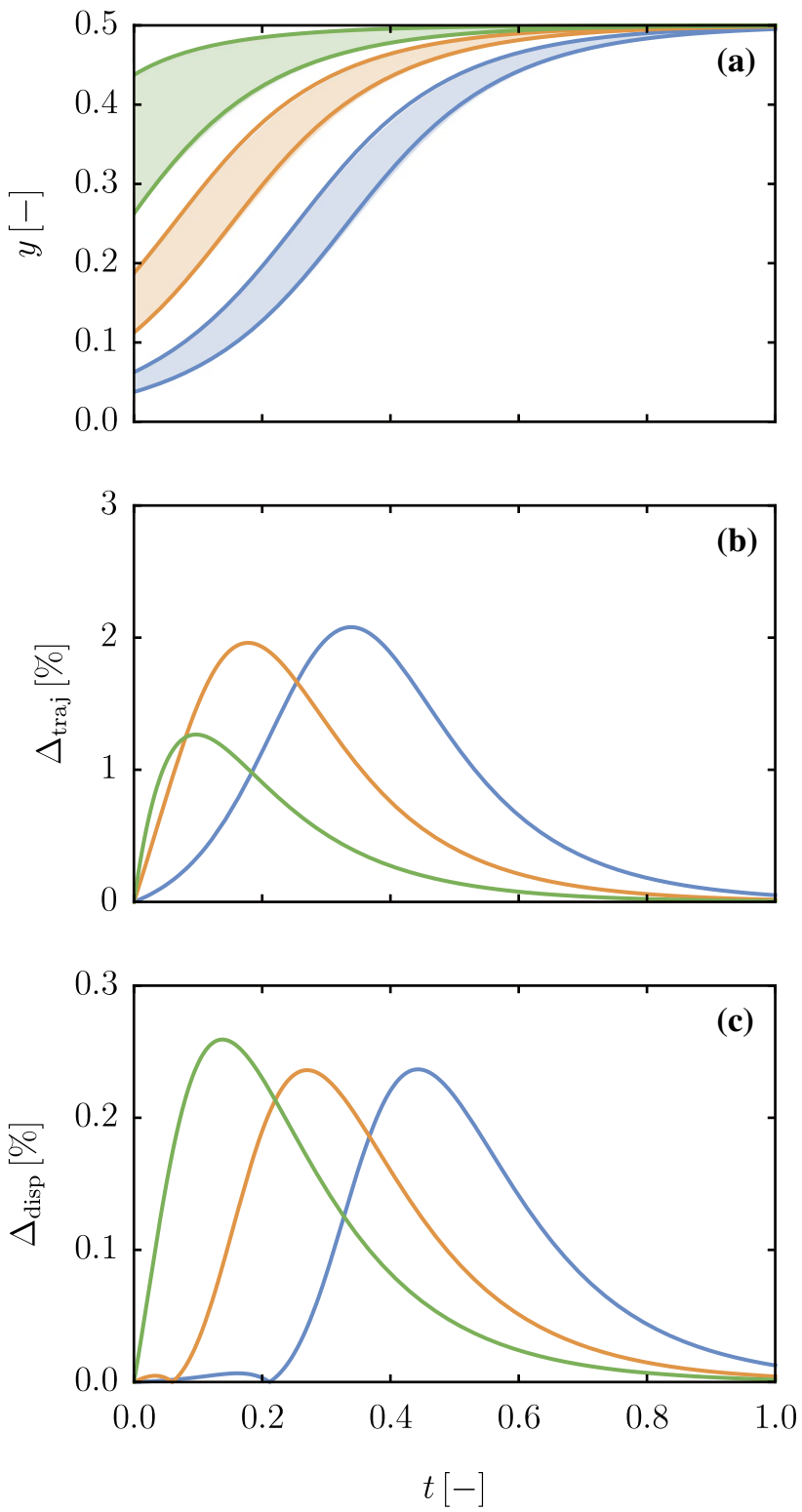

Fig. 2 (Color Online) Results for the model Eq. (18). a Bandwidth areas (colored areas) and dispersion bands $m^{y} \pm \frac{1}{2} \sqrt{s^{y y}}$ (lines) as function of the time $t$. b Distance between the average trajectories $\Delta_{\text {traj }}$ and $\mathbf{c}$ distance between the dispersion characteristics $\Delta_{\text {disp }}$ as function of the time $t$ corresponding to the cases in a. The initial conditions are: $y_{0}=0.05$ (blue), $y_{0}=0.15$ (orange), and $y_{0}=0.35$ (green). For all of the simulations it has $m_{0}^{r}=1, \quad s_{0}^{r r}=0, s_{0}^{y r}=0$, and $\sqrt{s_{0}^{y y}}=\mathrm{BW}(0)=50 \% y_{0}$

for the dispersion, where the factor 1 was included in the denominator to prevent divergent values for $\Delta_{\text {disp }}$, since the variance approaches zero for large $t$. These quantities are reported in Fig. 2b, c, respectively. As it can be seen from a practical point of view and in small perturbation cases, the two methods are equivalent with a maximum distance for 

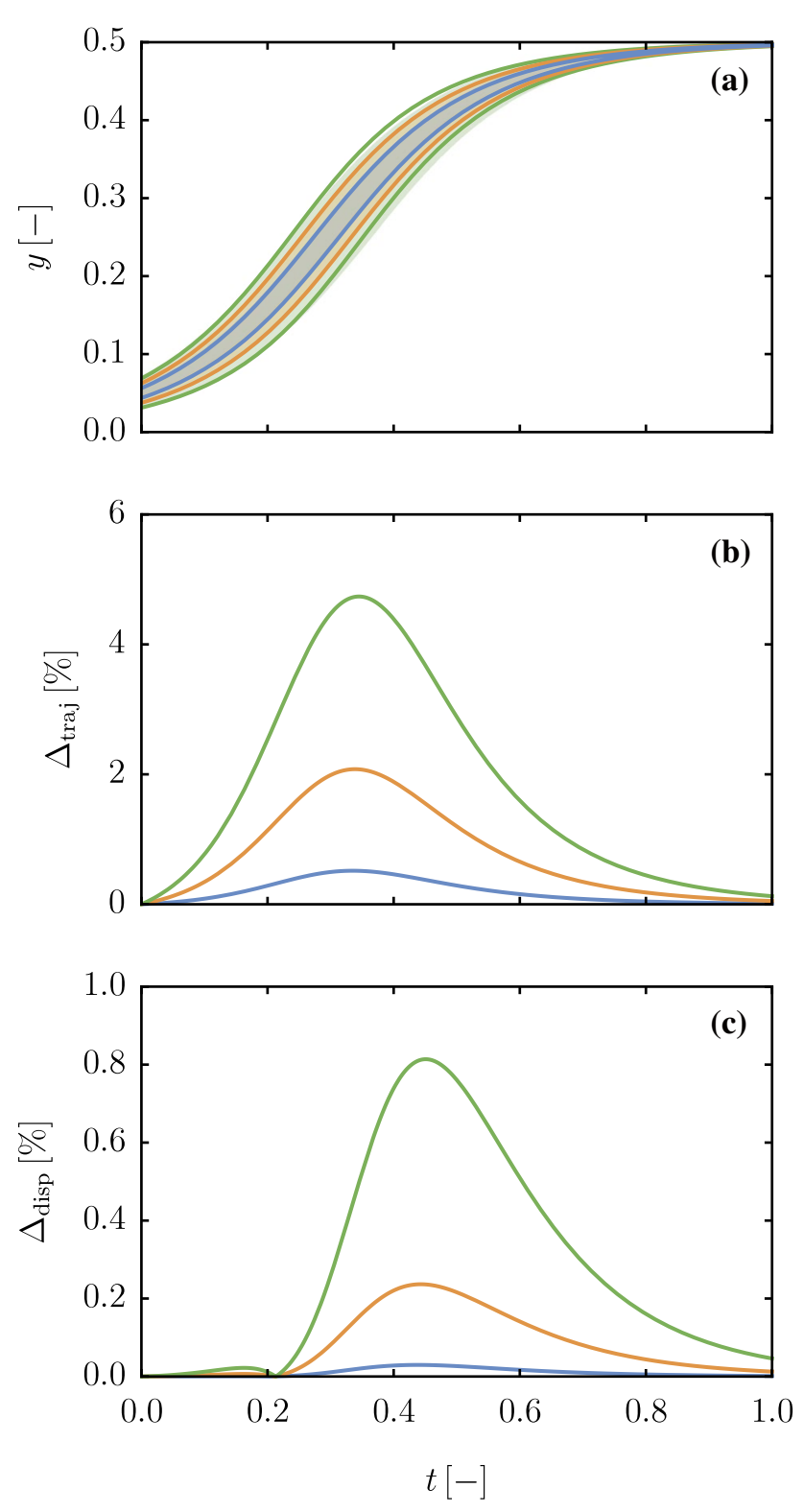

Fig. 3 (Color Online) Results for the model Eq. (18). a Bandwidth areas (colored areas) and dispersion bands $m^{y} \pm \frac{1}{2} \sqrt{s^{y y}}$ (lines) as function of the time $t$. b Distance between the average trajectories $\Delta_{\text {traj }}$ and $\mathbf{c}$ distance between the dispersion characteristics $\Delta_{\text {disp }}$ as function of the time $t$ corresponding to the cases in panel A. The initial conditions are for $y_{0}=0.05$ and: $\sqrt{s_{0}^{y y}}=25 \% y_{0}$ (blue), $\sqrt{s_{0}^{y y}}=50 \% y_{0}$ (orange), $\sqrt{s_{0}^{y y}}=75 \% y_{0}$ (green). For all of the simulations it has $m_{0}^{r}=1$ and $s_{0}^{r r}=0$

the trajectories less than $3 \%$ and less than $0.3 \%$ for the maximum distance in the dispersion.

Figure 3 shows an analogous comparison. In this case the different curves are for the same inlet condition $y_{0}$ and different values of the initial dispersion or bandwidth. It is noticeable that for bandwidth less than $100 \% y_{0}$ the two methods give yet very close results, i.e., within $6 \%$ in the trajectory distance $\Delta_{\text {traj }}$ and within $1 \%$ in the dispersion distance $\Delta_{\text {disp }}$. Let us consider now the case when the initial perturbation occurs in the spatial and radius components, that is when $\sqrt{s_{0}^{r r}} \neq 0$. In this case one can force the methodology proposed by Yang et al. by considering the perturbation $\boldsymbol{u}_{0}=\sqrt{s_{0}^{x x}} \hat{\boldsymbol{y}}+\sqrt{s_{0}^{r r}} \hat{\boldsymbol{r}}$, where both the $y$-direction and $r$-direction are meant in the coordinate-parameter space. This specific perturbation was chosen by considering the "best" and "worst" cases: the trajectory starting closer to the wall features a radius smaller than the average, and the trajectory starting far from the wall features a radius bigger than the average.

Figure 4 shows the comparison between the two methods in terms of bandwidth areas and dispersion bands (panel A) for different values of the inlet condition $y_{0}$, an initial perturbation equals to $50 \% y_{0}$, radius $m^{r}=1$, and initial perturbation for the radius equals $\sqrt{s_{0}^{r r}}=0.1$. In this plot it is easily visible that the two methods do not give exactly the same results; indeed, observing the behaviors of $\Delta_{\text {traj }}$ (panel B) and $\Delta_{\text {disp }}$ (panel C), it can be appreciated that the distances are larger than in the univariate cases, especially for the distance in the dispersion characteristics $\Delta_{\text {disp }}$ (see Sect. 4 for a discussion).

Finally, the comparison between the separation indicators is illustrated. Here the use of the parameter $\alpha$ is necessary as in Yang's method the separation efficiency is defined for particles with the same radius, i.e., $R$ in Eq. (13) in Yang et al. (2018). Figure 5 shows the results in terms of the Yang's indicators (Yang et al. 2018) and those above defined. Panel A shows the superposition of the two "dispersed" particle streams and the comparison between the bandwidth areas and the dispersion bands, which results very close to each other as in the limit of small perturbations. Panel B shows Yang's ideal separation efficiency ISE and the resolution index RI, which have very distinct behaviors. Panel C shows the comparison of Yang's separation efficiency SE and the separation resolution SR. Also for these two indicators the behaviors are different. It is even more important to notice that the maximum in SE and SR does not coincide. Specifically, the separation resolution anticipates the position for the optimal "section" where the two particle streams are better separated (see Sect. 4 for a discussion). Therefore, in terms of separation indicators, the two methods give different results.

\section{Discussion}

The method used by Yang and Vitali is conceptually similar, but not identical to the method proposed by Garofalo. They give practically identical results in terms of bandwidth and 

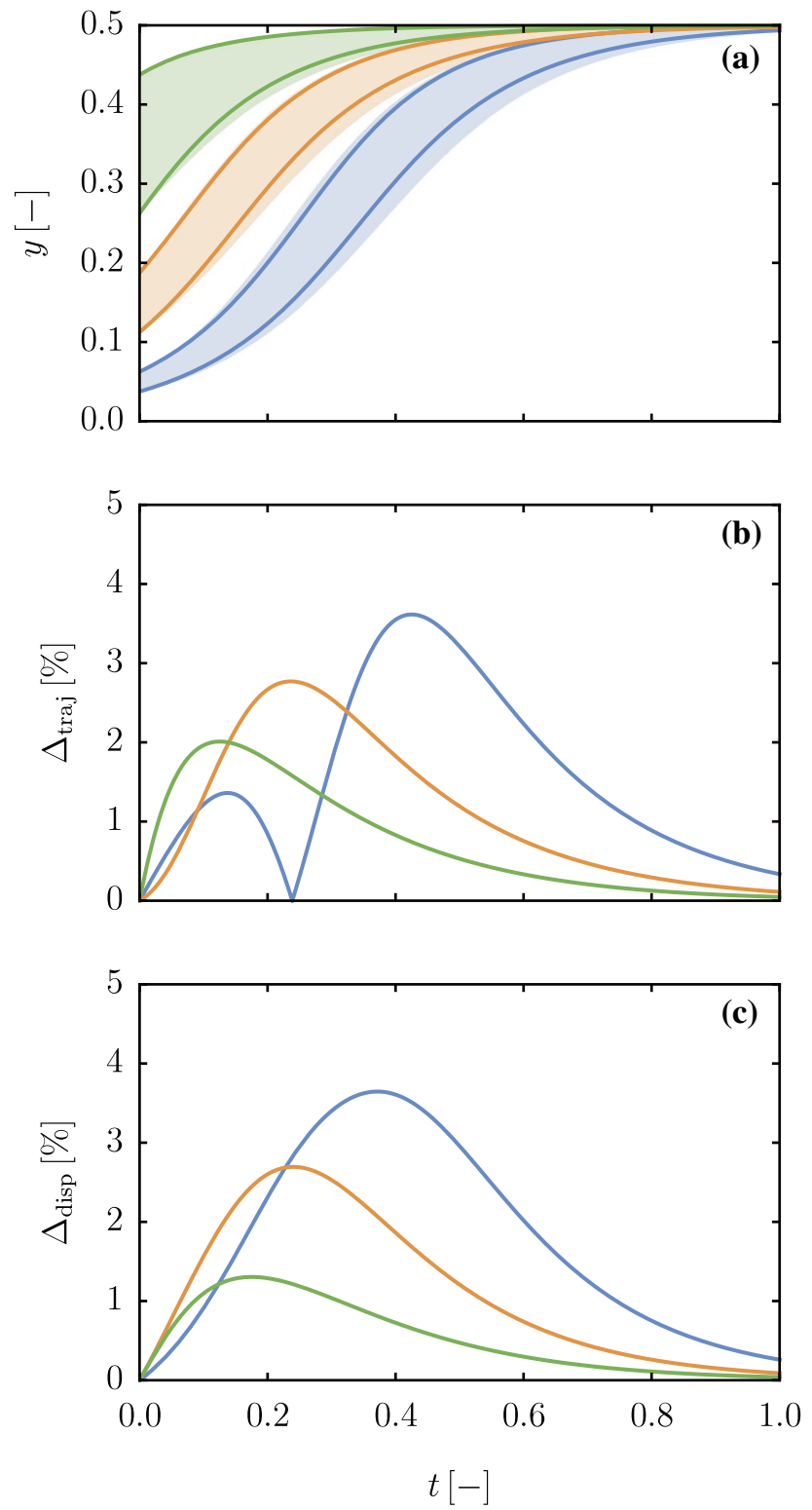

Fig. 4 (Color Online) Results for the model Eq. (18). a Bandwidth areas (colored areas) and dispersion bands $m^{y} \pm \frac{1}{2} \sqrt{s^{y y}}$ (lines) as function of the time $t$. b Distance between the average trajectories $\Delta_{\text {traj }}$ and $\mathbf{c}$ distance between the dispersion characteristics $\Delta_{\text {disp }}$ as function of the time $t$ corresponding to the cases in panel A. The initial conditions are: $y_{0}=0.05$ (blue), $y_{0}=0.15$ (orange), and $y_{0}=0.35$ (green). For all of the simulations it has $m_{0}^{r}=1, \sqrt{s_{0}^{r r}}=0.1, s_{0}^{y r}=0$, and $\sqrt{s_{0}^{y y}}=\mathrm{BW}(0)=50 \% y_{0}$

dispersion band in the case of univariate (single parameter) and small perturbation analysis. However, when the Yang's method is applied to multiparametric sensitivity analysis, as illustrated in Fig. 4, and when the indicators for the two methods are compared, the lack of statistical dispersion analysis gives different results.

In Yang's method, a distributed population with mean $\boldsymbol{m}$ and covariance matrix $s$ is represented just by two extremal
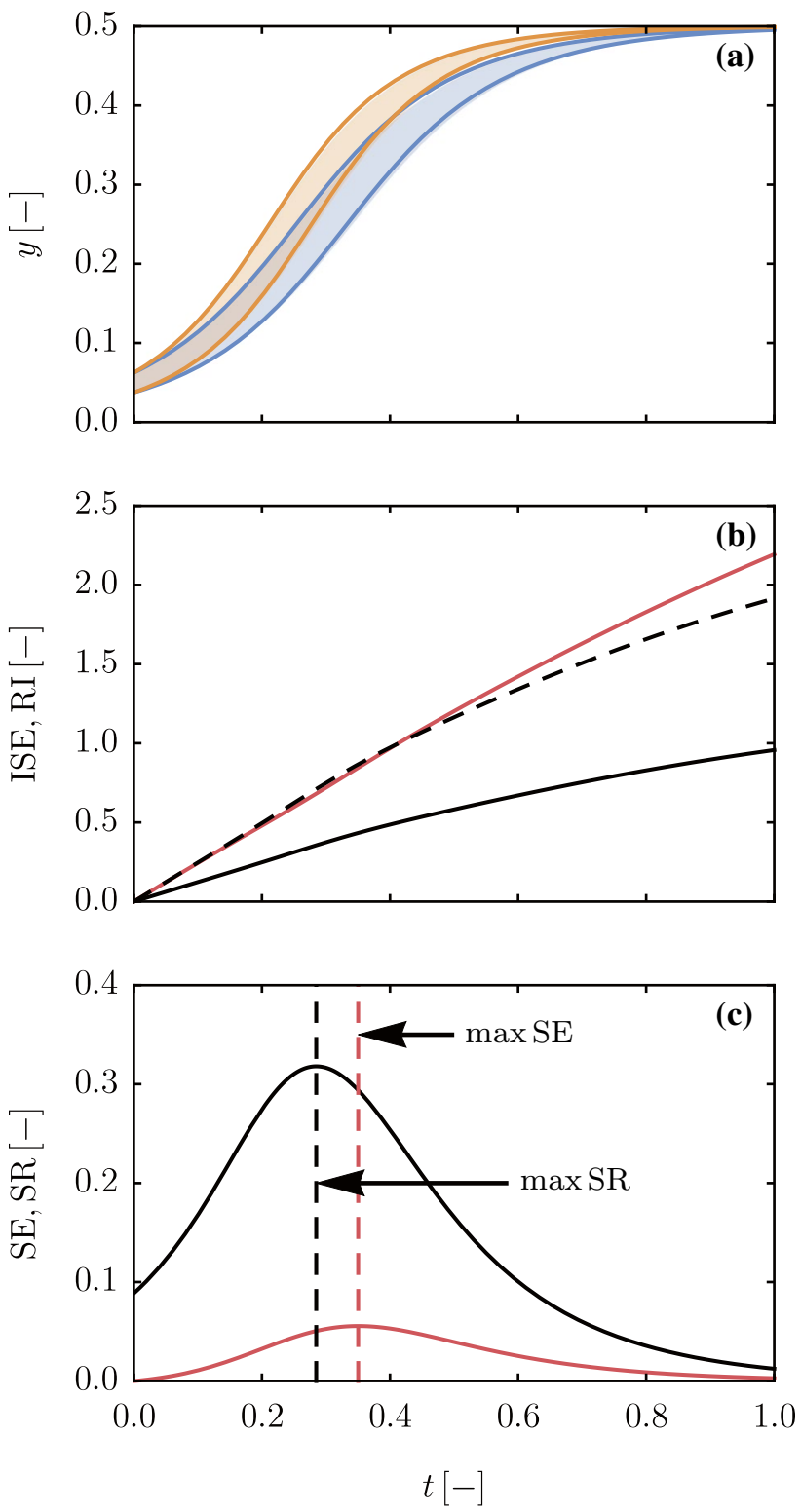

Fig. 5 (Color Online) a Bandwidth areas (colored areas), dispersion bands (lines), b ideal separation efficiency (red), resolution index (black, dashed is $2 \times \mathrm{RI}$ ), and (C) separation efficiency (black), separation resolution (red) as function of time $t$. The initial conditions are $y_{0}=0.05, m_{0}^{r}=1, s_{0}^{r r}=0, s_{0}^{y r}=0$, and $\sqrt{s_{0}^{y y}}=\mathrm{BW}(0)=50 \% y_{0}$. The values for $\alpha$ are $\alpha_{1}=1$ (blue) and $\alpha_{2}=1.2$ (orange)

values, and for that Yang's method assumes a different statistics for the sample. Figure 6 shows the representations of this fact in a simplified way. A particle population (dots) distributed in both space and parameter is represented by its mean and variance in statistics-based method (ellipse), while it is represented by extremal values (empty/filled circles) in the case of the kinematic-based method by Yang et al. Note also that in the case of Yang's method it must be established a priori what is the combination for the 


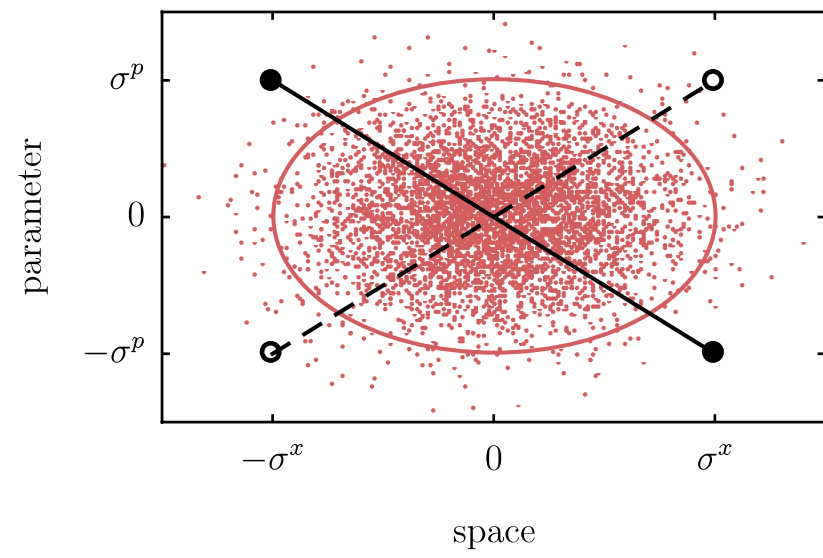

Fig. 6 (Color Online) Example of sample statistics for randomly picked particles with spatial dispersion $\sigma^{x}$ and parameter dispersion $\sigma^{p}$ (dots). The red ellipse is associated with the covariance matrix in Garofalo's method, the dots are associated with Yang's method and they represent the extremal values

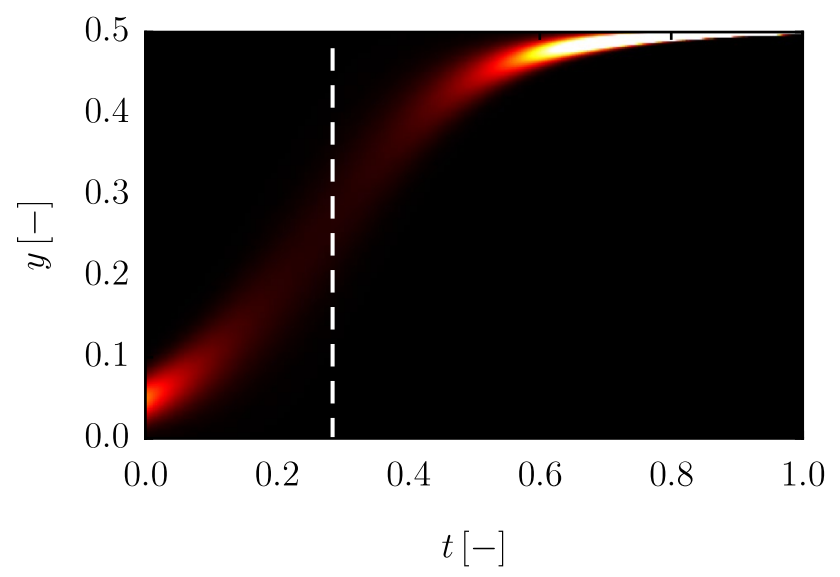

Fig. 7 (Color Online) Product of the spatial marginals for the case reported in Fig. 5 as function of the time $t$ and the cross-sectional position $y$. The vertical line corresponds to the max SR line depicted in Fig. 5c

"worst" and "best" cases, while in statistics-based method this is not necessary. It is even more important to recognize that in statistical dispersion analysis the parameter dispersion $s_{0}^{p p}$ is involved in the dynamics of spatial dispersion $s^{x x}$ by means of the cross-covariance $s^{x p}$ one-way coupling.

Another issue with Yang's method is that it does not provide information on the particle number density. The discrepancies observed between the information provided by SE and SR are a consequence of this lacking. Indeed, in the statistical method the product in the integral Eq. (14) gives a measure of the superposition of the two particle streams in terms of position, dispersion and particle number density of the two streams; see Fig. 7. Conversely, Yang's method takes into account only the position and the dispersion of the two particle streams. It would be possible to include the particle number density by randomly picking particles in the coordinate-parameter space and evolve the corresponding trajectories (Simon et al. 2017). However, as discussed in Garofalo (2018), this approach is computationally inconvenient, and Garofalo's method supersedes this type of simulations. Furthermore, the generalization of Yang's indicators is not straigthforward in the case of distributed populations.

Finally, the information provided by the particle number density (see Garofalo 2018) is essential when the characterization method aims (1) to quantify the separation efficiency, (2) to measure single-value property, and (3) to infer parameter histograms. Applications of the statistic-based method for acoustophoresis separations have been thoroughly investigated for arbitrary statistics in Garofalo (2018).

\section{Concluding remarks}

In this communication we harmonize the approach used by Yang and Vitali for the study of the parameters' influence on acoustophoretic separation with the statistic-based method in Garofalo (2014a, 2018). We recognize a partial equivalence of the two methods by (1) connecting the meanand-covariance dynamics with the perturbative approach adopted by Yang et al. for the case of small perturbation and (2) comparing the heuristic indicators they introduced with indicators derived by adopting the statistic approach. Furthermore, we addressed the limitations of a statistic-less method when applied to the characterization of processes in which distributed parameters are involved.

Ultimately, we can see that the methodology introduced by Yang et al. coincides with the method proposed by Garofalo when statistic-less and univariate small perturbations are considered. However, Yang's method does not extend straightforwardly in the case of multivariate parametric sensitivity analysis, and when the information of the particle number density is requested.

Open Access This article is distributed under the terms of the Creative Commons Attribution 4.0 International License (http://creativeco mmons.org/licenses/by/4.0/), which permits unrestricted use, distribution, and reproduction in any medium, provided you give appropriate credit to the original author(s) and the source, provide a link to the Creative Commons license, and indicate if changes were made.

\section{References}

Garofalo F (2014a) Analytical characterization of particle kynematics and transverse dispersion in free-flow acoustophoretic devices. Microfluid Nanofluid 18(3):367-382 
Garofalo F (2014b) Free-flow acoustofluidic devices: kinematics, crosssectional dispersion and particle ensemble correlations. ASME 2014 3rd global congress on nanoengineering for medicine and biology NEMB2014-93092. https://www.researchgate.net/publi cation/259962346_Free-flow_acoustofluidic_devices_kinem atics_cross-sectional_dispersion_and_particle_ensemble_corre lations_Presentation

Garofalo F (2017) Modeling particle populations in acoustophoretic manipulation. CBMS the 14th conference on acoustofluidics, San Diego (CA), August 28-29, 2017. https://www.researchgate.net/ publication/259962346_Free-flow_acoustofluidic_devices_kinem atics_cross-sectional_dispersion_and_particle_ensemble_corre lations_Presentation

Garofalo F (2018) Quantifying acoustophoretic separation of microparticle populations by mean-and-covariance dynamics for Gaussians in mixture models. arXiv: 1802.09790
Simon G, Andrade MAB, Reboud J, Marques-Hueso J, Desmulliez MPY, Cooper JM, Riehle MO, Bernassau AL (2017) Particle separation by phase modulated surface acoustic waves. Biomicrofluidics 11:0541155

Vitali V, Yang T, Minzioni P (2018) Separation efficiency maximization in acoustofluidic systems: study of the sample launch-position. RSC Adv 8:38955

Yang T, Vitali V, Minzioni P (2018) Acoustofluidic separation: impact of microfluidic system design and of sample properties. Microfluid Nanofluidic 22:44-56

Publisher's Note Springer Nature remains neutral with regard to jurisdictional claims in published maps and institutional affiliations. 